\title{
H-ras Antisense Oligodeoxynucleotide ISIS 2503
}

National Cancer Institute

\section{Source}

National Cancer Institute. H-ras Antisense Oligodeoxynucleotide ISIS 2503. NCI

Thesaurus. Code C2024.

A synthetic oligodeoxynucleotide. Functioning as an anti-sense agent, it hybridizes to the translation initiation region of the human mRNA for the oncogene H-Ras. ISIS 2503 selectively inhibits the expression of H-Ras, and may inhibit the growth of some Rasdependent tumor cells. ( $\mathrm{NCIO4)}$ 